\title{
Isolation of different erythromycin-resistance Streptococcus pneumoniae phenotypes in Piemonte Region
}

Valeria Allizond', Giuliana Banche', Janira Roana', Nicolò Li Vigni², Narcisa Mandras', Daniela Scalas', Vivian Tullio', Nicola A. Carlone', Dianella Savoia ${ }^{3}$, Anna Barbui ${ }^{4}$, Anna Maria Cuffini'

'Dipartimento di Sanità Pubblica e Microbiologia, Università degli Studi di Torino

2Laboratorio Analisi e Microbiologia, ASL 12, Biella

${ }^{3}$ Dipartimento di Scienze Cliniche e Biologiche, Università degli Studi di Torino

${ }^{4}$ Laboratorio di Microbiologia, ASO S. Giovanni Battista, Torino

Isolation of different erythromycin-resistance Streptococcus pneumoniae phenotypes in Piemonte Region

Key words: Streptococcus pneumoniae, Erythromycin resistance, Resistance phenotypes

\section{SUMMARY}

The frequency of erythromycin resistance in Streptococcus pneumoniae isolates from human specimens, in different Piemonte Region hospitals between 2005-2007, has been studied. Erythromycin-susceptible isolates were I37//98 and erythromycin-resistant 6I//98. Among resistant S. pneumoniae isolates 26/6I belonged to constitutive resistance phenotype, $26 / 6 \mathrm{I}$ to $M$ phenotype and $9 / 6 \mathrm{I}$ to inducible resistance phenotype.

Received December 19, 2007

Accepted January 18, 2008

\section{INTRODUZIONE}

Streptococcus pneumoniae è un ospite frequente delle prime vie respiratorie da dove, in presenza di concause predisponenti (infezioni respiratorie virali, traumi toracici e/o cranici, insufficienza cardiaca, età avanzata, ecc.), può raggiungere le vie respiratorie profonde provocando la polmonite. Dalle prime vie aeree il batterio può diffondersi e determinare vari quadri patologici tra cui batteriemie, endocarditi, meningiti, sinusiti ed otiti medie $(1,6,12,14)$.

Nonostante i $\beta$-lattamici siano considerati farmaci di elezione, una valida alternativa in caso di allergia o resistenza è rappresentata dai macrolidi il cui utilizzo clinico è stato recentemente ristretto a causa della crescente incidenza di resistenza batterica anche a questi antibiotici $(8,9,13,14)$. Due sono i meccanismi responsabili della resistenza ai macrolidi in $S$. pneumoniae: il primo è dovuto all'efflusso attivo dell'antibiotico attraverso la membrana cellulare mediante pompa protonica (fenotipo M, geni mef) e, in tal caso, la resistenza si esplica verso macrolidi con 14-15 atomi (eritromicina, claritromicina, azitromicina); il secondo è dovuto a mutazioni, in proteine ribosomiali o nei geni per il 23S rRNA, legate alla pro- duzione di una metilasi, con conseguente blocco del legame di macrolidi, lincosamidi e streptogramina $\mathrm{B}$ al ribosoma batterico (fenotipo $\mathrm{MLS}_{\mathrm{B}}$, geni erm) $(2,3,5)$. L'espressione di quest'ultimo fenotipo di resistenza può essere di tipo costitutivo (fenotipo cMLS ) od inducibile (fenotipo $\mathrm{iMLS}_{\mathrm{B}}$ ). Nella resistenza cMLS $\mathrm{S}_{\mathrm{B}}$ il batterio produce una metilasi sempre attiva, mentre per quella

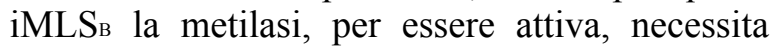
della presenza del macrolide che agisca da induttore $(6,8,11)$.

\section{PRESENTAZIONE DELLO STUDIO}

Nel presente studio è stato valutato il fenotipo di resistenza ai macrolidi su 198 ceppi di S. pneumoniae isolati, nel biennio 2005-2007, da differenti campioni biologici (tabella 1) provenienti da pazienti afferenti agli Ospedali San Giovanni Battista di Torino, San Luigi Gonzaga di Orbassano e degli Infermi di Biella. I campioni venivano seminati su agar Columbia con aggiunta del 5\% di sangue di montone (Biolife Italiana, Milano, Italia); dopo incubazione in $\mathrm{CO}_{2}$ al 5\% per $18-24$ ore alla temperatura di $37^{\circ} \mathrm{C}$, le colonie con i caratteristici aloni di $\alpha$-emolisi sono state 
isolate e successivamente identificate mediante $\mathrm{i}$ test di sensibilità all'optochina, della solubilità nella bile, ed infine con il sistema APIStrept (bioMérieux Italia, Roma, Italia).

La determinazione dei fenotipi di resistenza ai macrolidi è stata eseguita mediante il test del triplo disco secondo la metodica di Giovanetti E. et al (5). Sono stati utilizzati dischetti commerciali di eritromicina $(15 \mu \mathrm{g})$, clindamicina $(2 \mu \mathrm{g})$ e josamicina $[(30 \mu \mathrm{g})$ Oxoid, Basingstoke, Hampshire, England]. I dischetti sono stati posti ad una distanza di 15-20 mm l'uno dall'altro su una piastra di Mueller Hinton agar addizionato con il 5\% di sangue di montone (Oxoid), inoculata con una sospensione batterica con torbidità equivalente allo standard $0.5 \mathrm{McF}$ arland. Dopo $18-24$ ore d'incubazione a $37^{\circ} \mathrm{C}$ con il $5 \%$ di $\mathrm{CO}_{2}$, sono stati valutati gli aloni d'inibizione. La crescita batterica attorno all'eritromicina e la presenza di aloni d'inibizione attorno alla clindamicina ed alla josamicina caratterizza il fenotipo M; l'assenza di aloni d'inibizione attorno ai tre dischetti indica un fenotipo di resistenza costitutivo $\left(\mathrm{cMLS}_{\mathrm{B}}\right)$; la crescita attorno all'eritromicina e presenza di aloni smussati, $D$-shaped, attorno alla clindamicina ed alla josami-

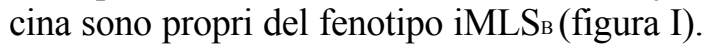

Tabella I. Numero di ceppi e percentuali di isolamento di S. pneumoniae da differenti campioni biologici

\begin{tabular}{lcc}
\hline CAMPIONI & $\begin{array}{c}\text { NUMERO DI CEPPI } \\
\text { (TOTALE 198) }\end{array}$ & $\%$ \\
\hline Emocoltura & 87 & 43.9 \\
\hline Escreato & 33 & 16.7 \\
\hline Tracheo bronco aspirato & 18 & 9.1 \\
\hline Liquor & 12 & 6.1 \\
\hline Tampone auricolare & 9 & 4.5 \\
\hline Tampone congiuntivale & 6 & 3.0 \\
\hline Essudato nasale & 6 & 3.0 \\
\hline Liquido pleurico & 3 & 1.5 \\
\hline Tampone vaginale & 3 & 1.5 \\
\hline Vari & 21 & 10.7 \\
\hline
\end{tabular}

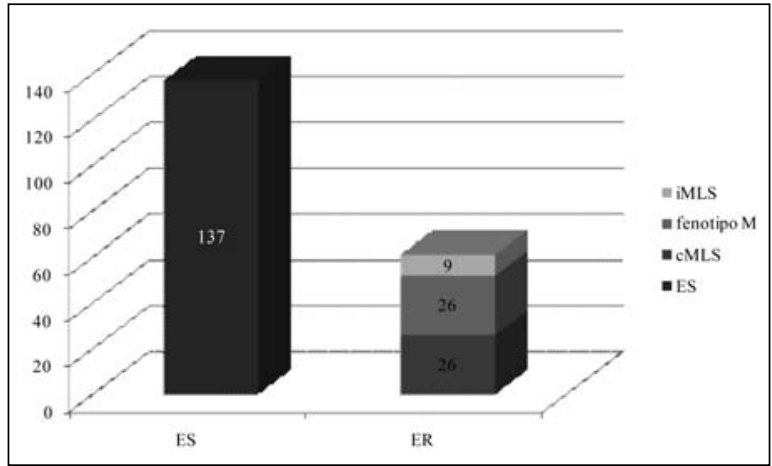

Fenotipo $M=$ fenotipo con pompa di efflusso; cMLS= resistenza costitutiva; $\mathrm{iMLS}=$ resistenza inducibile

Figura II. Distribuzione degli isolati di S. pneumoniae eritromicino-sensibili (ES) ed eritromicino-resistenti $(E R)$ con i differenti fenotipi di resistenza: $c M L S$, fenotipo $M$ ed $i M L S$

\section{CONCLUSIONI}

S. pneumoniae deve essere costantemente preso in considerazione nelle ipotesi eziologiche che riguardano tutte le infezioni respiratorie non solo per la frequenza con cui questo microrganismo è coinvolto ma anche per la forte tendenza ad acquisire, attraverso ricombinazione genetica, tratti di antibiotico-resistenza multipli che coinvolgono con grande frequenza anche $\beta$-lattamici e macrolidi (13). La gravità del fenomeno è molto variabile in funzione della diversa pressione selettiva operata dalle modalità d'impiego degli antibiotici da parte di ciascuna comunità medica nonché dalla circolazione di particolari cloni sul territorio $(9,12,13)$. In Italia un'attenta e continuativa opera di monitoraggio ha rilevato una consistente e consolidata incidenza (intorno al 25-30\%) di resistenza a macrolidi $(4,10,13)$.

I ceppi di $S$. pneumoniae oggetto di questo studio provengono da differenti campioni biologici: la maggior parte di essi è stata isolata da emocoltura $(43.9 \%)$, escreato $(16.7 \%)$ e tracheo-bronco

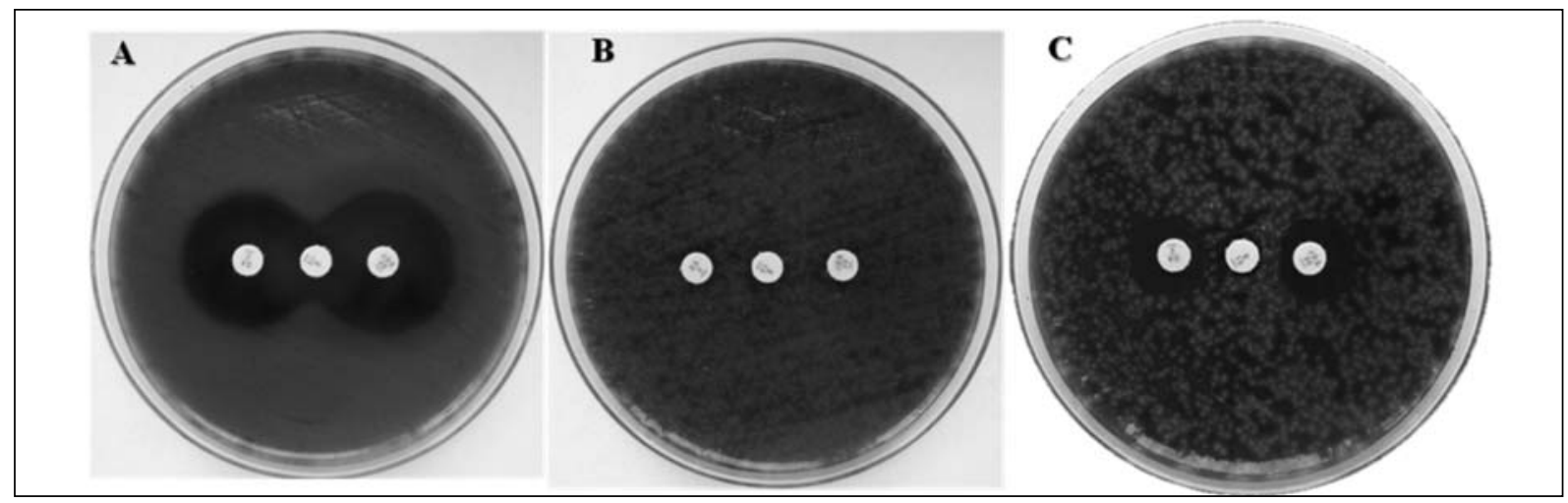

Fenotipo $M=$ fenotipo con pompa di efflusso; $c M L S=$ resistenza costitutiva; iMLS= resistenza inducibile

Figura I. Fenotipi di S. pneumoniae ER - fenotipo $M(\boldsymbol{A}), c M L S(B), i M L S(C)$ - come determinato con il test del triplo disco: al centro di ciascuna piastra è posta l'eritromicina (E; I5 $\mu \mathrm{g})$, sulla sinistra la clindamicina (DA; $2 \mu \mathrm{g})$, sulla destra la josamicina (JOS; $30 \mu \mathrm{g})$ 
aspirato (9.1\%) come riportato in tabella 1 . Dei 198 ceppi di S. pneumoniae isolati, il 69.2\% risulta eritromicino-sensibile (ES) ed il 30.8\% eritromicino-resistente (ER). Nell'ambito degli ER, sulla base del test del triplo disco, 26 ceppi $(42.6 \%)$ sono resistenti ai macrolidi per attivazione di un sistema di pompa di efflusso (fenotipo M) mentre 35 ceppi (57.4\%) mostrano un fenotipo di resistenza MLS $_{\mathrm{B}}$ : in particolare il $42.6 \%$ degli isolati è produttore di una metilasi sempre attiva (fenotipo $\mathrm{cMLS}_{\mathrm{B}}$ ) mentre il 14.8\% necessita del macrolide per l'attivazione della metilasi (fenotipo $\mathrm{iMLS}_{\mathrm{B}}$; figure I e II).

In conclusione, con il presente lavoro, si intende enfatizzare come la continua sorveglianza della distribuzione dei fenotipi di eritromicino-resistenza in $S$. pneumoniae, nelle differenti aree geografiche, sia essenziale al fine di instaurare la più appropriata terapia antibiotica. Infatti, è necessario avvalersi di una strategia terapeutica che escluda l'impiego di tutti gli antibiotici facenti parte del gruppo MLS $_{\text {в }}$ nel caso di isolamento di pneumococchi con fenotipi $\mathrm{cMLS}_{\text {в }}$ ed $\mathrm{iMLS}_{\mathrm{в}}$ i quali presentano una resistenza allargata nei confronti di tutto lo spettro delle molecole appartenenti a questo gruppo. Al contrario, l'utilizzo di un macrolide a 16 atomi (josamicina e rokitamicina) o di un ketolide (telitromicina) è suggerito qualora vengano isolati ceppi di $S$. pneumoniae con fenotipo a pompa di efflusso in quanto notoriamente resistenti ai macrolidi a 14 e 15 atomi $(7,10,15)$.

\section{BIBLIOGRAFIA}

1. Drago L, De Vecchi E, Nicola L, Legnani D, Gismondo MR. Kinetic bactericidal activity of telithromycin, azithromycin and clarithromycin against respiratory pathogens. APMIS 2005; 113: 655-63.

2. Farrell DJ, File TM, Jenkins SG. Prevalence and antibacterial susceptibility of mef(A)-positive macrolideresistant Streptococcus pneumoniae over 4 years (2000 to 2004) of the PROTEKT US Study. J Clin Microbiol 2007; 45: 290-3.

3. Felmingham D, Canton R, Jenkins SG. Regional trends in beta-lactam, macrolide, fluoroquinolone and telithromycin resistance among Streptococcus pneumoniae isolates 2001-2004. J Infect 2007; 55: 111-8.
4. Giovanetti E, Montanari MP, Marchetti F, Varaldo PE. In vitro activity of ketolides telithromycin and HMR 3004 against italian isolates of Streptococcus pyogenes and Streptococcus pneumoniae with different erythromycin susceptibility. J Antimicrob Chemother 2000; 46: 905-8.

5. Giovanetti E, Montanari MP, Mingoia M, Varaldo PE. Phenotypes and genotypes of erythromycin-resistant Streptococcus pyogenes strains in Italy and heterogeneity of inducibly resistant strains. Antimicrob Agents Chemother 1999; 43: 1935-40.

6. Harimaya A, Yokota S, Sato K, Yamazaki N, Himi T, Fujii N. High prevalence of erythromycin resistance and macrolide-resistance genes, mef $\mathrm{A}$ and erm $\mathrm{B}$, in Streptococcus pneumoniae isolates from the upper respiratory tracts of children in the Sapporo district, Japan. J Infect Chemother 2007; 13: 219-23.

7. Ioannidou $\mathrm{S}$, Tassios PT, Zachariadou L, et al. In vitro activity of telithromycin (HMR 3647) against Greek Streptococcus pyogenes and Streptococcus pneumoniae clinical isolates with different macrolide susceptibilities. Clin Microbiol Infect 2003; 9: 704-7.

8. Leclercq R. Mechanisms of resistance to macrolides and lincosamides: nature of the resistance elements and their clinical implications. Clin Infect Dis 2002; 34: 482-92.

9. Marchese A, Schito GC. Resistance patterns of lower respiratory tract pathogens in Europe. Int $\mathrm{J}$ Antimicrob Agents 2000; 16: S25-9. Review.

10. Mazzariol A, Koncan R, Vitali LA, Cornaglia G. Activities of 16-membered ring macrolides and telithromycin against different genotypes of erythromycin-susceptible and erythromycin-resistant Streptococcus pyogenes and Streptococcus pneumoniae. J Antimicrob Chemother 2007; 59: 1171-6.

11. Montanari MP, Mingoia M, Giovanetti E, Varaldo PE. Differentiation of resistance phenotypes among erythromycin-resistant Pneumococci. J Clin Microbiol 2001; 39: 1311-5

12. Neuman MI, Kelley M, Harper MB, File TM Jr, Camargo CA Jr. EMNet Investigators. Factors associated with antimicrobial resistance and mortality in pneumococcal bacteremia. J Emerg Med 2007; 32: 349-57.

13. Shibl AM. Patterns of macrolide resistance determinants among $S$. pyogenes and $S$. pneumoniae isolates in Saudi Arabia. J Int Med Res 2005; 33: 349-55.

14. Tarallo L, Tancredi F, Schito G, Marchese A, Bella A. Italian Pneumonet Group (Società Italiana Pediatria and Associazione Italiana Studio Antimicrobici e Resistenze). Active surveillance of Streptococcus pneumoniae bacteremia in Italian children. Vaccine. 2006; 24: 6938-43.

15. Zhanel GG, Walters M, Noreddin A, et al. The ketolides: a critical review. Drugs 2002; 62: 1771804. 